\title{
Taxonomy of the order Mononegavirales: update 2017
}

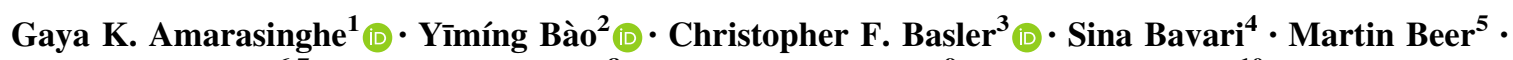

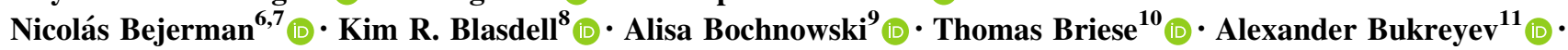

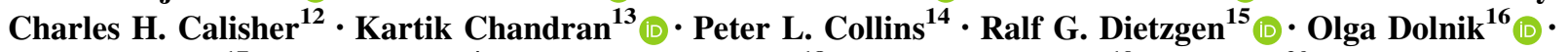
Ralf Dürrwald $^{17} \cdot$ John M. Dye $^{4}$ (D) Andrew J. Easton ${ }^{18}$ (D) Hideki Ebihara ${ }^{19} \cdot$ Qi Fang $^{20} \cdot$ Pierre Formenty $^{21}$ (D) Ron A. M. Fouchier ${ }^{22}$ (D) Elodie Ghedin ${ }^{23}$ (D) Robert M. Harding $^{24}$ (D) Roger Hewson ${ }^{25}$ - Colleen M. Higgins ${ }^{26,27}$. Jian Hong ${ }^{28} \cdot$ Masayuki Horie $^{29}$ (D) Anthony P. James ${ }^{24}$ (D) Dàohóng Jiāng ${ }^{30}$ - Gary P. Kobinger ${ }^{31}$ (D) Hideki Kondo ${ }^{32} \cdot$ Gael Kurath $^{33}$ (D) Robert A. Lamb ${ }^{34,35} \cdot$ Benhur Lee $^{36}$ - Eric M. Leroy ${ }^{37} \cdot$ Ming Li $^{26,27}$. Andrea Maisner $^{16}$ (i) $\cdot$ Elke Mühlberger $^{38}$ - Sergey V. Netesov ${ }^{39}$ (i) $\cdot$ Norbert Nowotny $^{40,41}$ (D) Jean L. Patterson $^{42}$. Susan L. Payne ${ }^{43}$ (1) Janusz T. Paweska ${ }^{44}$ (1) Michael N. Pearson ${ }^{45} \cdot$ Rick E. Randall $^{46} \cdot$ Peter A. Revill $^{24,47} \cdot$ Bertus K. Rima ${ }^{48} \cdot$ Paul Rota $^{49} \cdot$ Dennis Rubbenstroth $^{50} \cdot$ Martin Schwemmle $^{50} \cdot$ Sophie J. Smither $^{51}$. Qisheng Song ${ }^{52} \cdot$ David M. Stone $^{53}$ (1) Ayato Takada $^{54} \cdot$ Calogero Terregino $^{55}$ (1) Robert B. Tesh ${ }^{11}$. Keizo Tomonaga $^{56} \cdot$ Noël Tordo $^{57,66} \cdot$ Jonathan S. Towner $^{58} \cdot$ Nikos Vasilakis $^{11}$ (1) Viktor E. Volchkov ${ }^{59}$. Victoria Wahl-Jensen $^{60}$ (D) Peter J. Walker ${ }^{61}$ (D) Beibei Wang $^{20} \cdot$ David Wang $^{62} \cdot$ Fei Wang $^{20} \cdot$ Lin-Fa Wang $^{63} \cdot$

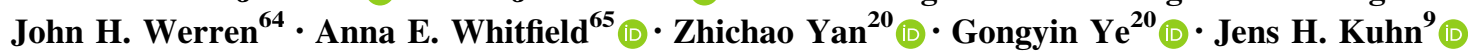

Received: 19 February 2017/Accepted: 26 February 2017/Published online: 7 April 2017

(C) Springer-Verlag Wien (Outside the USA) 2017

\begin{abstract}
In 2017, the order Mononegavirales was expanded by the inclusion of a total of 69 novel species. Five new rhabdovirus genera and one new nyamivirus genus were established to harbor 41 of these species, whereas the remaining new species were assigned to already established genera. Furthermore, non-Latinized
\end{abstract}

binomial species names replaced all paramyxovirus and pneumovirus species names, thereby accomplishing application of binomial species names throughout the entire order. This article presents the updated taxonomy of the order Mononegavirales as now accepted by the International Committee on Taxonomy of Viruses (ICTV).

Members of the International Committee on Taxonomy of Viruses (ICTV) Bornaviridae, Filoviridae, Mononegavirales, Nyamiviridae, Paramyxoviridae, and Rhabdoviridae Study Groups who co-authored this manuscript are listed in "Acknowledgements".

Jens H. Kuhn

kuhnjens@mail.nih.gov

1 Department of Pathology and Immunology, Washington University School of Medicine, St. Louis, MO, USA

2 Information Engineering Branch, National Center for Biotechnology Information, National Library of Medicine, National Institutes of Health, Bethesda, MD, USA

3 Center for Microbial Pathogenesis, Institute for Biomedical Sciences, Georgia State University, Atlanta, GA, USA

4 United States Army Medical Research Institute of Infectious Diseases, Fort Detrick, Frederick, MD, USA

5 Institute of Diagnostic Virology, Friedrich-Loeffler-Institut, Greifswald-Insel Riems, Germany
Instituto de Patología Vegetal, Centro de Investigaciones Agropecuarias, Instituto Nacional de Tecnología Agropecuaria, Córdoba, Argentina

7 Consejo Nacional de Investigaciones Científicas y Técnicas, Buenos Aires, Argentina

8 CSIRO Health and Biosecurity, Australian Animal Health Laboratory, Geelong, Victoria, Australia

9 Integrated Research Facility at Fort Detrick (IRF-Frederick), Division of Clinical Research (DCR), National Institute of Allergy and Infectious Diseases (NIAID), National Institutes of Health (NIH), B-8200 Research Plaza, Fort Detrick, Frederick, MD 21702, USA

10 Center for Infection and Immunity, Mailman School of Public Health, Columbia University, New York, NY, USA 


\section{Introduction}

The virus order Mononegavirales was established in 1991 to accommodate related viruses with nonsegmented, linear, single-stranded negative-sense RNA genomes. These viruses were initially assigned to three mononegavirus families: Filoviridae, Paramyxoviridae, and Rhabdoviridae [32, 33]. The families Bornaviridae and Nyamiviridae joined the order in 1996 [34] and 2014 [1, 25], respectively, followed by the families Mymonaviridae, Pneumoviridae, and Sunviridae in 2016 [2]. The order was continuously amended in 1995 [7], 1997 [35], 2000 [36], 2005 [37], 2011 [17], and 2016 [2]. In 2016, the Study Groups of the International Committee on Taxonomy of Viruses (ICTV) responsible for the taxonomy of the order and its eight families assigned unclassified mononegaviruses to existing or novel taxa and continued efforts to streamline order nomenclature. Here we present the changes that were (re)proposed via official ICTV taxonomic proposals (TaxoProps) at http://www.ictvon line.org/ in 2016 and that were accepted by the ICTV Executive Committee. These changes are official ICTV taxonomy as of 2017 .

\section{Taxonomic changes at the order level}

No changes were made at the order level.

11 Department of Pathology, The University of Texas Medical Branch, Galveston, TX, USA

12 Arthropod-Borne and Infectious Diseases Laboratory, College of Veterinary Medicine and Biomedical Sciences, Colorado State University, Fort Collins, CO, USA

13 Department of Microbiology and Immunology, Albert Einstein College of Medicine, Bronx, New York, USA

14 Respiratory Viruses Section, Laboratory of Infectious Diseases, National Institute of Allergy and Infectious Diseases, National Institutes of Health, Bethesda, MD, USA

15 Queensland Alliance for Agriculture and Food Innovation, The University of Queensland, St. Lucia, QLD, Australia

16 Institute of Virology, Philipps University Marburg, Marburg, Germany

17 MICROMUN GmbH, Greifswald, Germany

18 School of Life Sciences, University of Warwick, Coventry, UK

19 Department of Molecular Medicine, Mayo Clinic, Rochester, MN, USA

20 State Key Laboratory of Rice Biology and Ministry of Agriculture Key Lab of Molecular Biology of Crop Pathogens and Insects, Institute of Insect Sciences, Zhèjiāng University, Hángzhōu, China

\section{Taxonomic changes at the family level}

\section{Bornaviridae}

The family Bornaviridae remains monogeneric but was expanded in 2017 by one species (Mammalian 2 bornavirus) for the newly discovered variegated squirrel bornavirus 1 [23] (TaxoProp 2016.013aM.A.v1.Bornavirus_sp).

\section{Filoviridae}

No changes were made to this family.

\section{Mymonaviridae}

No changes were made to this family.

\section{Nyamiviridae}

The family Nyamiviridae was expanded to include a third genus (Peropuvirus) including the new species Pteromalus puparum peropuvirus for a virus recently discovered in parasitoid wasps, Pteromalus puparum negative-strand RNA virus 1 [45] (TaxoProp 2016.015a-dM.A.v1.Peropuvirus).

\section{Paramyxoviridae}

The non-Latinized binomial species name format [40] was applied throughout the family Paramyxoviridae (TaxoProp

21 World Health Organization, Geneva, Switzerland

22 Department of Viroscience, Erasmus Medical Center, Rotterdam, The Netherlands

23 Department of Biology, Center for Genomics and Systems Biology, New York University, New York, NY, USA

24 Centre for Tropical Crops and Biocommodities, Queensland University of Technology, Brisbane, QLD, Australia

25 Public Health England, Porton Down, Wiltshire, Salisbury, UK

26 Institute of Applied Ecology, School of Science, Auckland University of Technology, Auckland, New Zealand

27 AUT Roche Diagnostic Laboratory, Auckland University of Technology, Auckland, New Zealand

28 Analysis Center of Agrobiology and Environmental Sciences and Institute of Agrobiology and Environmental Sciences, Zhèjiāng University, Hángzhōu, China

29 Hakubi Center for Advanced Research, Kyoto University, Kyoto, Japan

30 State Key Laboratory of Agricultural Microbiology, The Provincial Key Lab of Plant Pathology of Húběi Province, College of Plant Science and Technology, Huázhōng Agricultural University, Wǔhàn, China 
2016.011aM.A.v2.Paramyxoviridae_spren). In addition, the genus Avulavirus was expanded by one novel species, Avian avulavirus 13, for avian paramyxovirus 13 discovered in geese [19, 24, 47] (TaxoProp 2016.001a,bM.A.v2.Avulavirus_spren). The genus Rubulavirus was expanded by 10 species to accommodate two long-known viruses (e.g., Menangle virus, Tioman virus) and eight newly discovered bat viruses (bat mumps virus, Achimota viruses 1 and 2, Sosuga virus, Teviot virus, Tuhoko viruses 1-3) [3, 5, 11, 12, 16, 26] (TaxoProp 2015.016aM.A.v3.Rubulavirus_10sp). Finally, the previous species name Newcastle disease virus was changed to Avian avulavirus 1 to reflect the fact that "Newcastle disease virus" is but one strain of the member virus of this species, avian paramyxovirus 1 (APMV-1) [46] (TaxoProp 2016.001a,bM.A.v2.Avulavirus_spren).

\section{Pneumoviridae}

The non-Latinized binomial species name format [40] was applied throughout the family Pneumoviridae (TaxoProp 2016.012aM.A.v1.Pneumoviridae_spren).

31 Department of Microbiology, Immunology and Infectious Diseases Université Laval, Quebec City, Canada

32 Institute of Plant Science and Resources, Okayama University, Kurashiki, Japan

33 US Geological Survey Western Fisheries Research Center, Seattle, Washington, USA

34 Department of Molecular Biosciences, Northwestern University, Evanston, IL, USA

35 Howard Hughes Medical Institute, Northwestern University, Evanston, IL, USA

36 Department of Microbiology, Icahn School of Medicine at Mount Sinai, New York, NY, USA

37 Centre International de Recherches Médicales de Franceville, Institut de Recherche pour le Développement, Franceville, Gabon

38 Department of Microbiology and, National Emerging Infectious Diseases Laboratories, Boston University School of Medicine, Boston, MA, USA

39 Novosibirsk State University, Novosibirsk, Novosibirsk Oblast, Russia

40 Institute of Virology, University of Veterinary Medicine, Vienna, Austria

41 Department of Basic Medical Sciences, College of Medicine, Mohammed Bin Rashid University of Medicine and Health Sciences, Dubai, United Arab Emirates

\section{Rhabdoviridae}

The most extensive changes were introduced into the family Rhabdoviridae. The new genus Almendravirus was created to include five new species (TaxoProp 2016.002adM.A.v3.Almendravirus) to accommodate mosquito viruses discovered from 2009-2013 (Arboretum virus, Balsa virus, Coot Bay virus, Puerto Almendras virus, and Rio Chico virus) [13, 43, 44]. The new genus Curiovirus, including four new species, was created for unclassified dipteran viruses known since the 1970s-80s (Curionopolis virus, Iriri virus, Itacaiunas virus, Rochambeau virus) (TaxoProp 2016.003a-dM.A.v3.Curiovirus) [14, 15, 44]. Twelve mostly dipteran viruses (Hart Park virus, Gray Lodge virus, Joinjakaka virus, Kamese virus, La Joya virus, Landjia virus, Manitoba virus, Marco virus, Mosqueiro virus, Mossuril virus, Ord River virus, and Parry Creek virus), isolated in the 1950 s to late 1970 s, were classified into twelve new species in the new genus $\mathrm{Ha}$ pavirus. The previously free-floating rhabdovirus species Flanders virus, Ngaingan virus, and Wongabel virus were moved into the genus Hapavirus and renamed accordingly (Flanders hapavirus, Ngaingan hapavirus, and Wongabel
42 Department of Virology and Immunology, Texas Biomedical Research Institute, San Antonio, TX, USA

43 Department of Veterinary Pathobiology, College of Veterinary Medicine and Biomedical Sciences, Texas A\&M University, College Station, TX, USA

44 Center for Emerging and Zoonotic Diseases, National Institute for Communicable Diseases of the National Health Laboratory Service, Sandringham, Johannesburg, Gauteng, South Africa

45 School of Biological Sciences, The University of Auckland, Auckland, New Zealand

46 Biomedical Sciences Research Complex, University of St. Andrews, St. Andrews, Scotland, UK

47 Victorian Infectious Diseases Reference Laboratory, Doherty Institute of Infection and Immunity, Melbourne, Victoria, Australia

48 Centre for Experimental Medicine, School of Medicine, Dentistry and Biomedical Sciences, The Queen's University of Belfast, Belfast, Northern Ireland, UK

49 National Center for Immunization and Respiratory Diseases, Centers for Disease Control and Prevention, Atlanta, Georgia, USA

50 Institute for Virology, Medical Center, University of Freiburg, Faculty of Medicine, University of Freiburg, Freiburg, Germany 
hapavirus, respectively) [4, 21, 44] (TaxoProp 2016.005agM.A.v4.Hapavirus). The largest newly created rhabdovirus genus is Ledantevirus. This genus includes 14 new species for Barur virus, Fikirini virus, Fukuoka virus, Kern Canyon virus, Keuraliba virus, Kolente virus, Kumasi rhabdovirus, Le Dantec virus, Mount Elgon bat virus, Nkolbisson virus, Nishimuro virus, Oita virus, Wǔhàn louse fly virus 5 , and Yǒngjiā tick virus 2 , which were isolated from or detected in arthropods and/or mammals [6, 10, 18, 28, 38, 44] (TaxoProp 2016.006adM.A.v2.Ledantevirus). Finally, the novel genus Sripuvirus was created to include five new species for sandfly and lizard viruses (Almpiwar virus, Chaco virus, Niakha virus, Sena Madureira virus, and Sripur virus) [29, 41, 44] (TaxoProp 2016.007a-dM.A.v5.Sripuvirus).

The existing genus Cytorhabdovirus was expanded by inclusion of the novel species Colocasia bobone disease-associated cytorhabdovirus for a plant virus first described in 1973 in the taro plant (Colocasia esculenta)] [22] (TaxoProp 2016.017aM.A.v1.Cytorhabdovirus_sp). The genus Ephemerovirus was enlarged by three species to accommodate the long-known bovid viruses Kimberley and Koolpinyah, and the mosquito-borne Malakal and Yata viruses [8, 9] (TaxoProp 2016.004aM.A.v2.Ephemerovirus_3sp). The recently described, possibly human, Bas-Congo virus and Ekpoma viruses 1 and 2, and the biting midge-borne Sweetwater Branch virus were classified into four new species of the genus

51 CBR Division, Defence Science and Technology Laboratory, Porton Down, Salisbury, Wiltshire, UK

52 Division of Plant Sciences, College of Agriculture, Food and Natural Resources, University of Missouri, Columbia, Missouri, USA

53 Centre for Environment, Fisheries and Aquaculture Science, Weymouth, Dorset, UK

54 Division of Global Epidemiology, Hokkaido University Research Center for Zoonosis Control, Sapporo, Japan

55 Istituto Zooprofilattico Sperimentale delle Venezie, Department of Comparative Biomedical Sciences, National/ OIE Reference Laboratory for Newcastle Disease and Avian Influenza, FAO Reference Centre for Animal Influenza and Newcastle Disease, OIE Collaborating Centre for Diseases at the Human-Animal Interface, Legnaro, Padova, Italy

56 Institute for Frontier Life and Medical Sciences (inFront), Kyoto University, Kyoto, Japan

57 Institut Pasteur, Unité des Stratégies Antivirales, WHO Collaborative Centre for Viral Haemorrhagic Fevers and Arboviruses, OIE Reference Laboratory for RVFV and CCHFV, Paris, France

58 Viral Special Pathogens Branch, Division of HighConsequence Pathogens Pathology, National Center for Emerging and Zoonotic Infectious Diseases, Centers for Disease Control and Prevention, Atlanta, Georgia, USA
Tibrovirus [20, 27, 39, 44] (TaxoProp 2016.008aM.A.v3.Tibrovirus_4sp). Klamath virus, discovered in 1962 in a vole, was assigned to a novel species in the genus Tupavirus [44] (TaxoProp 2016.009aM.A.v2.Tupavirus_sp), and seven new species were added to the genus Vesiculovirus for American bat vesiculovirus (isolated from bats in 2008) and Jurona virus, Malpais Spring virus, Morreton virus, Perinet virus, Radi virus, and Yug Bogdanovac virus (isolated from mosquitoes or sandflies in 1962-1986) [22, 30, 31, 42, 44] (TaxoProp 016.010aM.A.v3.Vesiculovirus_7sp).

Finally, the existing species names Oncorhynchus 1 novirhabdovirus (formerly Infectious hematopoietic necrosis virus) and Oncorhynchus 2 novirhabdovirus (formerly Viral hemorrhagic septicemia virus) were changed to Salmonid novirhabdovirus and Piscine novirhabdovirus, respectively, to be more reflective of the host spectrum of their members. (2016.018aM.A.v1.Novirhabdovirus_spren).

\section{Sunviridae}

In 2017, no changes were made to this family.

\section{Summary}

A summary of the current, ICTV-accepted taxonomy of the order Mononegavirales is presented in Table 1.

59 Molecular Basis of Viral Pathogenicity, CIRIINSERM U1111 - CNRS UMR5308, Université de Lyon, Université Claude Bernard Lyon 1, Ecole Normale Supérieure de Lyon, Lyon, France

60 National Biodefense Analysis and Countermeasures Center, Fort Detrick, Frederick, MD, USA

61 School of Biological Sciences, University of Queensland, St. Lucia, QLD, Australia

62 Departments of Molecular Microbiology and Pathology and Immunology, Washington University School of Medicine, St. Louis, MO, USA

63 Programme in Emerging Infectious Diseases, Duke-NUS Medical School, Singapore, Singapore

64 Department of Biology, University of Rochester, Rochester, NY, USA

65 Plant Pathology, Kansas State University, Manhattan, KS, USA

66 Institut Pasteur de Guinée, Conakry, Guinea 
Table 1 ICTV-accepted taxonomy of the order Mononegavirales as of 2017

\begin{tabular}{|c|c|c|}
\hline Genus & Species & Virus (Abbreviation) \\
\hline \multicolumn{3}{|l|}{ Family Bornaviridae } \\
\hline \multirow[t]{16}{*}{ Bornavirus } & Elapid 1 bornavirus & Loveridge's garter snake virus 1 (LGSV-1) \\
\hline & Mammalian 1 bornavirus* & Borna disease virus 1 (BoDV-1) \\
\hline & & Borna disease virus 2 (BoDV-2) \\
\hline & Mammalian 2 bornavirus & variegated squirrel bornavirus 1 (VSBV-1) \\
\hline & Passeriform 1 bornavirus & canary bornavirus $1(\mathrm{CnBV}-1)$ \\
\hline & & canary bornavirus $2(\mathrm{CnBV}-2)$ \\
\hline & & canary bornavirus $3(\mathrm{CnBV}-3)$ \\
\hline & Passeriform 2 bornavirus & estrildid finch bornavirus 1 (EsBV-1) \\
\hline & Psittaciform 1 bornavirus & parrot bornavirus $1(\mathrm{PaBV}-1)$ \\
\hline & & parrot bornavirus $2(\mathrm{PaBV}-2)$ \\
\hline & & parrot bornavirus 3 (PaBV-3) \\
\hline & & parrot bornavirus $4(\mathrm{PaBV}-4)$ \\
\hline & & parrot bornavirus 7 (PaBV-7) \\
\hline & Psittaciform 2 bornavirus & parrot bornavirus $5(\mathrm{PaBV}-5)$ \\
\hline & Waterbird 1 bornavirus & aquatic bird bornavirus 1 (ABBV-1) \\
\hline & & aquatic bird bornavirus $2(\mathrm{ABBV}-2)$ \\
\hline \multicolumn{3}{|l|}{ Family Filoviridae } \\
\hline Cuevavirus & Lloviu cuevavirus* & Lloviu virus (LLOV) \\
\hline \multirow[t]{5}{*}{ Ebolavirus } & Bundibugyo ebolavirus & Bundibugyo virus (BDBV) \\
\hline & Reston ebolavirus & Reston virus (RESTV) \\
\hline & Sudan ebolavirus & Sudan virus (SUDV) \\
\hline & Taï Forest ebolavirus & Taï Forest virus (TAFV) \\
\hline & Zaire ebolavirus* & Ebola virus (EBOV) \\
\hline \multirow[t]{2}{*}{ Marburgvirus } & Marburg marburgvirus* & Marburg virus (MARV) \\
\hline & & Ravn virus (RAVV) \\
\hline \multicolumn{3}{|l|}{ Family Mymonaviridae } \\
\hline Sclerotimonavirus & Sclerotinia sclerotimonavirus* & Sclerotinia sclerotiorum negative-stranded RNA virus 1 (SsNSRV-1) \\
\hline \multicolumn{3}{|l|}{ Family Nyamiviridae } \\
\hline \multirow[t]{3}{*}{ Nyavirus } & Midway nyavirus & Midway virus (MIDWV) \\
\hline & Nyamanini nyavirus* & Nyamanini virus (NYMV) \\
\hline & Sierra Nevada nyavirus & Sierra Nevada virus (SNVV) \\
\hline Peropuvirus & Pteromalus puparum peropuvirus* & Pteromalus puparum negative-strand RNA virus 1 (PpNSRV-1) \\
\hline Socyvirus & Soybean cyst nematode socyvirus* & soybean cyst nematode virus $1(\mathrm{SbCNV}-1)$ \\
\hline \multicolumn{3}{|c|}{ Family Paramyxoviridae } \\
\hline Aquaparamyxovirus & Salmon aquaparamyxovirus* & Atlantic salmon paramyxovirus (AsaPV) \\
\hline \multirow[t]{13}{*}{ Avulavirus } & Avian avulavirus $1 *$ & avian paramyxovirus $1(\mathrm{APMV}-1)^{1}$ \\
\hline & Avian avulavirus 2 & avian paramyxovirus 2 (APMV-2) \\
\hline & Avian avulavirus 3 & avian paramyxovirus $3(\mathrm{APMV}-3)$ \\
\hline & Avian avulavirus 4 & avian paramyxovirus 4 (APMV-4) \\
\hline & Avian avulavirus 5 & avian paramyxovirus 5 (APMV-5) \\
\hline & Avian avulavirus 6 & avian paramyxovirus 6 (APMV-6) \\
\hline & Avian avulavirus 7 & avian paramyxovirus 7 (APMV-7) \\
\hline & Avian avulavirus 8 & avian paramyxovirus 8 (APMV-8) \\
\hline & Avian avulavirus 9 & avian paramyxovirus 9 (APMV-9) \\
\hline & Avian avulavirus 10 & avian paramyxovirus 10 (APMV-10) \\
\hline & Avian avulavirus 11 & avian paramyxovirus 11 (APMV-11) \\
\hline & Avian avulavirus 12 & avian paramyxovirus 12 (APMV-12) \\
\hline & Avian avulavirus 13 & avian paramyxovirus 13 (APMV-13) \\
\hline Ferlavirus & Reptilian ferlavirus* & Fer-de-Lance virus $\left(\right.$ FDLV) ${ }^{2}$ \\
\hline Henipavirus & Cedar henipavirus & Cedar virus (CedV) \\
\hline
\end{tabular}


Table 1 continued

\begin{tabular}{|c|c|c|}
\hline Genus & Species ${ }^{\top}$ & Virus (Abbreviation) \\
\hline & Ghanaian bat henipavirus & Kumasi virus $(\mathrm{KV})^{3}$ \\
\hline & Hendra henipavirus* & Hendra virus $(\mathrm{HeV})$ \\
\hline & Mojiang henipavirus & Mòjiāng virus (MojV) \\
\hline & Nipah henipavirus & Nipah virus (NiV) \\
\hline \multirow[t]{7}{*}{ Morbillivirus } & Canine morbillivirus & canine distemper virus (CDV) \\
\hline & Cetacean morbillivirus & cetacean morbillivirus (CeMV) \\
\hline & Feline morbillivirus & feline morbillivirus (FeMV) \\
\hline & Measles morbillivirus* & measles virus $(\mathrm{MeV})$ \\
\hline & Small ruminant morbillivirus & peste-des-petits-ruminants virus (PPRV) \\
\hline & Phocine morbillivirus & phocine distemper virus (PDV) \\
\hline & Rinderpest morbillivirus & rinderpest virus (RPV) \\
\hline \multirow[t]{5}{*}{ Respirovirus } & Bovine respirovirus 3 & bovine parainfluenza virus 3 (BPIV-3) \\
\hline & Human respirovirus 1 & human parainfluenza virus 1 (HPIV-1) \\
\hline & Human respirovirus 3 & human parainfluenza virus 3 (HPIV-3) \\
\hline & Porcine respirovirus 1 & porcine parainfluenza virus 1 (PPIV-1) \\
\hline & Murine respirovirus* & Sendai virus $(\mathrm{SeV})^{4}$ \\
\hline \multirow[t]{18}{*}{ Rubulavirus } & Achimota rubulavirus 1 & Achimota virus 1 (AchPV-1) \\
\hline & Achimota rubulavirus 2 & Achimota virus 2 (AchPV-2) \\
\hline & Bat mumps rubulavirus & bat mumps virus $(\mathrm{BMV})^{5}$ \\
\hline & Canine rubulavirus & parainfluenza virus $5(P I V-5)^{6}$ \\
\hline & Human rubulavirus 2 & human parainfluenza virus 2 (HPIV-2) \\
\hline & Human rubulavirus 4 & human parainfluenza virus 4a (HPIV-4a) \\
\hline & & human parainfluenza virus 4b (HPIV-4b) \\
\hline & Mapuera rubulavirus & Mapuera virus (MapV) \\
\hline & Menangle rubulavirus & Menangle virus (MenPV) \\
\hline & Mumps rubulavirus* & mumps virus $(\mathrm{MuV})$ \\
\hline & Porcine rubulavirus & La Piedad Michoacán Mexico virus (LPMV) ${ }^{7}$ \\
\hline & Simian rubulavirus & simian virus 41 (SV-41) \\
\hline & Sosuga rubulavirus & Sosuga virus \\
\hline & Teviot rubulavirus & Teviot virus (TevPV) \\
\hline & Tioman rubulavirus & Tioman virus (TioPV) \\
\hline & Tuhoko rubulavirus 1 & Tuhoko virus 1 (ThkPV-1) \\
\hline & Tuhoko rubulavirus 2 & Tuhoko virus 2 (ThkPV-2) \\
\hline & Tuhoko rubulavirus 3 & Tuhoko virus 3 (ThkPV-3) \\
\hline \multicolumn{3}{|l|}{ Family Pneumoviridae } \\
\hline \multirow[t]{2}{*}{ Metapneumovirus } & Avian metapneumovirus* & avian metapneumovirus $(\mathrm{AMPV})^{8}$ \\
\hline & Human metapneumovirus & human metapneumovirus (HMPV) \\
\hline \multirow[t]{4}{*}{ Orthopneumovirus } & Bovine orthopneumovirus & bovine respiratory syncytial virus (BRSV) \\
\hline & Human orthopneumovirus* & human respiratory syncytial virus A2 (HRSV-A2) \\
\hline & & human respiratory syncytial virus B1 (HRSV-B1) \\
\hline & Murine orthopneumovirus & murine pneumonia virus (MPV) \\
\hline \multicolumn{3}{|l|}{ Family Rhabdoviridae } \\
\hline \multirow[t]{5}{*}{ Almendravirus } & Arboretum almendravirus & Arboretum virus (ABTV) \\
\hline & Balsa almendravirus & Balsa virus (BALV) \\
\hline & Coot Bay almendravirus & Coot Bay virus (CBV) \\
\hline & Puerto Almendras almendravirus* & Puerto Almendras virus (PTAMV) \\
\hline & Rio Chico almendravirus & Rio Chico virus (RCHV) \\
\hline \multirow[t]{4}{*}{ Curiovirus } & Curionopolis curiovirus* & Curionopolis virus (CURV) \\
\hline & Iriri curiovirus & Iriri virus (IRIRV) \\
\hline & Itacaiunas curiovirus & Itacaiunas virus (ITAV) \\
\hline & Rochambeau curiovirus & Rochambeau virus (RBUV) \\
\hline Cytorhabdovirus & Alfalfa dwarf cytorhabdovirus & alfalfa dwarf virus (ADV) \\
\hline
\end{tabular}


Table 1 continued

\begin{tabular}{|c|c|c|}
\hline$\overline{\text { Genus }}$ & Species ${ }^{\top}$ & Virus (Abbreviation) \\
\hline & Barley yellow striate mosaic cytorhabdovirus & barley yellow striate mosaic virus (BYSMV) \\
\hline & Broccoli necrotic yellows cytorhabdovirus & broccoli necrotic yellows virus (BNYV) \\
\hline & Colocasia bobone disease-associated cytorhabdovirus & Colocasia bobone disease-associated virus $(\mathrm{CBDaV})$ \\
\hline & Festuca leaf streak cytorhabdovirus & festuca leaf streak virus (FLSV) \\
\hline & Lettuce necrotic yellows cytorhabdovirus* & lettuce necrotic yellows virus (LNYV) \\
\hline & Lettuce yellow mottle cytorhabdovirus & lettuce yellow mottle virus (LYMoV) \\
\hline & Northern cereal mosaic cytorhabdovirus & northern cereal mosaic virus (NCMV) \\
\hline & Sonchus cytorhabdovirus 1 & sonchus virus (SonV) \\
\hline & Strawberry crinkle cytorhabdovirus & strawberry crinkle virus (SCV) \\
\hline & Wheat American striate mosaic cytorhabdovirus & wheat American striate mosaic virus (WASMV) \\
\hline \multirow[t]{2}{*}{ Dichorhavirus } & Coffee ringspot dichorhavirus & coffee ringspot virus (CoRSV) \\
\hline & Orchid fleck dichorhavirus* & orchid fleck virus $(\mathrm{OFV})^{9}$ \\
\hline \multirow[t]{9}{*}{ Ephemerovirus } & Adelaide River ephemerovirus & Adelaide River virus (ARV) \\
\hline & Berrimah ephemerovirus & Berrimah virus (BRMV) \\
\hline & Bovine fever ephemerovirus* & bovine ephemeral fever virus $(\mathrm{BEFV})^{10}$ \\
\hline & Kimberley ephemerovirus & Kimberley virus (KIMV) \\
\hline & & Malakal virus (MALV) \\
\hline & Koolpinyah ephemerovirus & Koolpinyah virus (KOOLV) \\
\hline & Kotonkan ephemerovirus & kotonkan virus (KOTV) \\
\hline & Obodhiang ephemerovirus & Obodhiang virus (OBOV) \\
\hline & Yata ephemerovirus & Yata virus (YATV) \\
\hline \multirow[t]{15}{*}{ Hapavirus } & Flanders hapavirus & Flanders virus (FLAV) \\
\hline & Hart Park hapavirus & Hart Park virus (HPV) \\
\hline & Gray Lodge hapavirus & Gray Lodge virus (GLOV) \\
\hline & Joinjakaka hapavirus & Joinjakaka virus (JOIV) \\
\hline & La Joya hapavirus & La Joya virus (LJV) \\
\hline & Kamese hapavirus & Kamese virus (KAMV) \\
\hline & Landjia hapavirus & Landjia virus (LANV = LJAV) \\
\hline & Manitoba hapavirus & Manitoba virus $(\mathrm{MANV}=\mathrm{MNTBV})$ \\
\hline & Marco hapavirus & Marco virus (MCOV) \\
\hline & Mosqueiro hapavirus & Mosqueiro virus (MQOV) \\
\hline & Mossuril hapavirus & Mossuril virus (MOSV) \\
\hline & Ngaingan hapavirus & Ngaingan virus (NGAV) \\
\hline & Ord River hapavirus & Ord River virus (ORV) \\
\hline & Parry Creek hapavirus & Parry Creek virus (PCV) \\
\hline & Wongabel hapavirus* & Wongabel virus (WONV) \\
\hline \multirow[t]{14}{*}{ Ledantevirus } & Barur ledantevirus & Barur virus (BARV) \\
\hline & Fikirini ledantevirus & Fikirini virus (FKRV) \\
\hline & Fukuoka ledantevirus & Fukuoka virus (FUKV) \\
\hline & Kern Canyon ledantevirus & Kern Canyon virus (KCV) \\
\hline & Keuraliba ledantevirus & Keuraliba virus (KEUV) \\
\hline & Kolente ledantevirus & Kolente virus (KOLEV) \\
\hline & Kumasi ledantevirus & Kumasi rhabdovirus (KRV) \\
\hline & Le Dantec ledantevirus* & Le Dantec virus (LDV) \\
\hline & Mount Elgon bat ledantevirus & Mount Elgon bat virus (MEBV) \\
\hline & Nkolbisson ledantevirus & Nkolbisson virus (NKOV) \\
\hline & Nishimuro ledantevirus & Nishimuro virus (NISV) ${ }^{11}$ \\
\hline & Oita ledantevirus & Oita virus (OITAV) \\
\hline & Wuhan ledantevirus & Wǔhàn louse fly virus 5 (WLFV-5) \\
\hline & Yongjia ledantevirus & Yǒngjiā tick virus 2 (YTV-2) \\
\hline \multirow[t]{3}{*}{ Lyssavirus } & Aravan lyssavirus & Aravan virus (ARAV) \\
\hline & Australian bat lyssavirus & Australian bat lyssavirus (ABLV) \\
\hline & Bokeloh bat lyssavirus & Bokeloh bat lyssavirus (BBLV) \\
\hline
\end{tabular}


Table 1 continued

\begin{tabular}{|c|c|c|}
\hline Genus & Species ${ }^{\top}$ & Virus (Abbreviation) \\
\hline & Duvenhage lyssavirus & Duvenhage virus (DUVV) \\
\hline & European bat 1 lyssavirus & European bat lyssavirus 1 (EBLV-1) \\
\hline & European bat 2 lyssavirus & European bat lyssavirus 2 (EBLV-2) \\
\hline & Ikoma lyssavirus & Ikoma lyssavirus (IKOV) \\
\hline & Irkut lyssavirus & Irkut virus (IRKV) \\
\hline & Khujand lyssavirus & Khujand virus (KHUV) \\
\hline & Lagos bat lyssavirus & Lagos bat virus (LBV) \\
\hline & Mokola lyssavirus & Mokola virus (MOKV) \\
\hline & Rabies lyssavirus* & rabies virus (RABV) \\
\hline & Shimoni bat lyssavirus & Shimoni bat virus (SHIBV) \\
\hline & West Caucasian bat lyssavirus & West Caucasian bat virus (WCBV) \\
\hline \multirow[t]{4}{*}{ Novirhabdovirus } & Hirame novirhabdovirus & Hirame rhabdovirus (HIRV) \\
\hline & Piscine novirhabdovirus & viral hemorrhagic septicemia virus $(\mathrm{VHSV})^{12}$ \\
\hline & Salmonid novirhabdovirus* & infectious hematopoietic necrosis virus (IHNV) \\
\hline & Snakehead novirhabdovirus & snakehead rhabdovirus (SHRV) \\
\hline \multirow[t]{11}{*}{ Nucleorhabdovirus } & Datura yellow vein nucleorhabdovirus & datura yellow vein virus (DYVV) \\
\hline & Eggplant mottled dwarf nucleorhabdovirus & eggplant mottled dwarf virus (EMDV) \\
\hline & Maize fine streak nucleorhabdovirus & maize fine streak virus (MSFV) \\
\hline & Maize Iranian mosaic nucleorhabdovirus & maize Iranian mosaic virus (MIMV) \\
\hline & Maize mosaic nucleorhabdovirus & maize mosaic virus (MMV) \\
\hline & Potato yellow dwarf nucleorhabdovirus* & potato yellow dwarf virus (PYDV) \\
\hline & Rice yellow stunt nucleorhabdovirus & rice yellow stunt virus (RYSV) \\
\hline & & rice transitory yellowing virus (RTYV) \\
\hline & Sonchus yellow net nucleorhabdovirus & sonchus yellow net virus (SYNV) \\
\hline & Sowthistle yellow vein nucleorhabdovirus & sowthistle yellow vein virus (SYVV) \\
\hline & Taro vein chlorosis nucleorhabdovirus & taro vein chlorosis virus (TaVCV) \\
\hline \multirow[t]{3}{*}{ Perhabdovirus } & Anguillid perhabdovirus & eel virus European X (EVEX) \\
\hline & Perch perhabdovirus* & perch rhabdovirus (PRV) \\
\hline & Sea trout perhabdovirus & lake trout rhabdovirus (LTRV) \\
\hline \multirow[t]{7}{*}{ Sigmavirus } & Drosophila affinis sigmavirus & Drosophila affinis sigmavirus (DAffSV) \\
\hline & Drosophila ananassae sigmavirus & Drosophila ananassae sigmavirus (DAnaSV) \\
\hline & Drosophila immigrans sigmavirus & Drosophila immigrans sigmavirus (DImmSV) \\
\hline & Drosophila melanogaster sigmavirus* & Drosophila melanogaster sigmavirus (DMelSV) \\
\hline & Drosophila obscura sigmavirus & Drosophila obscura sigmavirus (DObsSV) \\
\hline & Drosophila tristis sigmavirus & Drosophila tristis sigmavirus (DTriSV) \\
\hline & Muscina stabulans sigmavirus & Muscina stabulans sigmavirus (MStaSV) \\
\hline \multirow[t]{4}{*}{ Sprivivirus } & Carp sprivivirus* & spring viremia of carp virus (SVCV) \\
\hline & Pike fry sprivivirus & grass carp rhabdovirus (GrCRV) \\
\hline & & pike fry rhabdovirus (PFRV) \\
\hline & & tench rhabdovirus (TenRV) \\
\hline \multirow[t]{5}{*}{ Sripuvirus } & Almpiwar sripuvirus & Almpiwar virus (ALMV) \\
\hline & Chaco sripuvirus & Chaco virus $(\mathrm{CHOV})$ \\
\hline & Niakha sripuvirus* & Niakha virus (NIAV) \\
\hline & Sena Madueira sripuvirus & Sena Madueira virus (SMV) \\
\hline & Sripur sripuvirus & Sripur virus (SRIV) \\
\hline \multirow[t]{7}{*}{ Tibrovirus } & Bas-Congo tibrovirus & Bas-Congo virus (BASV) \\
\hline & Coastal Plains tibrovirus & Coastal Plains virus (CPV) \\
\hline & Ekpoma 1 tibrovirus & Ekpoma virus 1 (EKV-1) \\
\hline & Ekpoma 2 tibrovirus & Ekpoma virus 2 (EKV-2) \\
\hline & Sweetwater Branch tibrovirus & Sweetwater Branch virus (SWBV) \\
\hline & Tibrogargan tibrovirus* & Bivens Arm virus (BAV) \\
\hline & & Tibrogargan virus (TIBV) \\
\hline
\end{tabular}


Table 1 continued

\begin{tabular}{|c|c|c|}
\hline Genus & Species & Virus (Abbreviation) \\
\hline \multirow[t]{3}{*}{ Tupavirus } & Durham tupavirus* & Durham virus (DURV) \\
\hline & Klamath tupavirus & Klamath virus (KLAV) \\
\hline & Tupaia tupavirus & tupaia virus (TUPV) \\
\hline Varicosavirus & Lettuce big-vein associated varicosavirus* & lettuce big-vein associated virus $(\mathrm{LBVaV})^{13}$ \\
\hline \multirow[t]{16}{*}{ Vesiculovirus } & Alagoas vesiculovirus & vesicular stomatitis Alagoas virus (VSAV) \\
\hline & American bat vesiculovirus & American bat vesiculovirus (ABVV) \\
\hline & Carajas vesiculovirus & Carajás virus (CJSV) \\
\hline & Chandipura vesiculovirus & Chandipura virus (CHPV) \\
\hline & Cocal vesiculovirus & Cocal virus $(\mathrm{COCV})$ \\
\hline & Indiana vesiculovirus* & vesicular stomatitis Indiana virus (VSIV) \\
\hline & Isfahan vesiculovirus & Isfahan virus (ISFV) \\
\hline & Jurona vesiculovirus & Jurona virus (JURV) \\
\hline & Malpais Spring vesiculovirus & Malpais Spring virus (MSPV) \\
\hline & Maraba vesiculovirus & Maraba virus (MARAV) \\
\hline & Morreton vesiculovirus & Morreton virus (MORV) \\
\hline & New Jersey vesiculovirus & vesicular stomatitis New Jersey virus (VSNJV) \\
\hline & Perinet vesiculovirus & Perinet virus (PERV) \\
\hline & Piry vesiculovirus & Piry virus (PIRYV) \\
\hline & Radi vesiculovirus & Radi virus (RADV) \\
\hline & Yug Bogdanovac vesiculovirus & Yug Bogdanovac virus (YBV) \\
\hline Unassigned & Moussa virus & Moussa virus (MOUV) \\
\hline \multicolumn{3}{|c|}{ Family Sunviridae } \\
\hline Sunshinevirus & Reptile sunshinevirus $1 *$ & Sunshine Coast virus (SunCV) \\
\hline \multicolumn{3}{|l|}{ Unassigned } \\
\hline Anphevirus & Xincheng anphevirus* & Xīnchéng mosquito virus (XcMV) \\
\hline Arlivirus & Lishi arlivirus* & Líshí spider virus 2 (LsSV-2) \\
\hline Chengtivirus & Tacheng chengtivirus* & Tăchéng tick virus 6 (TcTV-6) \\
\hline Crustavirus & Wenzhou crustavirus* & Wēnzhōu crab virus 1 (WzCV-1) \\
\hline Wastrivirus & Sanxia wastrivirus* & Sānxiá water strider virus 4 (SxWSV-4) \\
\hline
\end{tabular}

* Asterisks denote type species. ${ }^{\uparrow}$ Please note that viruses are real objects that are assigned to concepts that are called taxa. Species, genera, families, and orders are taxa. The taxonomic changes listed here pertain to taxon name changes and do not affect virus names. Taxon names are always italicized and always begin with a capital letter. Virus names, on the other hand, are not italicized and are not capitalized, except if the name or a name component is a proper noun. For educational purposes, this column lists the virus names with their correct (lack of) capitalization ${ }^{1}$ Includes: Newcastle disease virus (NDV) and pigeon paramyxovirus 1 (PPMV-1); ${ }^{2}$ synonym: anaconda paramyxovirus; ${ }^{3}$ synonym: GHM74a virus; ${ }^{4}$ synonym: murine parainfluenza virus $1 ;{ }^{5}$ synonym: bat paramyxovirus; ${ }^{6}$ synonym: simian virus $5 ;{ }^{7}$ synonym: porcine rubulavirus; ${ }^{8}$ synonyms: avian pneumovirus, turkey rhinotracheitis virus; ${ }^{9}$ synonyms: citrus leprosis virus nuclear type, citrus necrotic spot virus; ${ }^{10}$ synonym: Tzipori virus; ${ }^{11}$ synonym: wild boar rhabdovirus 1 (WBRV1); ${ }^{12}$ synonyms: Egtved virus, Paralichthys olivaceus rhabdovirus; 13 synonym: tobacco stunt virus

Acknowledgements We thank Laura Bollinger (NIH/NIAID Integrated Research Facility at Fort Detrick, Frederick, MD, USA) for critically editing the manuscript.

Members of the International Committee on Taxonomy of Viruses (ICTV) Bornaviridae Study Group who coauthored this manuscript: Ralf Dürrwald, Masayuki Horie, Jens H. Kuhn, Norbert Nowotny, Susan L. Payne, Dennis Rubbenstroth, Thomas Briese, Martin Schwemmle, Keizo Tomonaga.

Members of the ICTV Filoviridae Study Group who coauthored this manuscript: Gaya K. Amarasinghe, Christopher F. Basler, Alexander Bukreyev, Kartik Chandran, Olga Dolnik, John M. Dye, Hideki Ebihara, Pierre Formenty, Roger Hewson, Gary P. Kobinger, Jens H. Kuhn, Eric M. Leroy, Elke Mühlberger, Sergey V. Netesov, Jean L. Patterson, Janusz T. Paweska, Sophie J. Smither, Ayato Takada, Jonathan S. Towner, Viktor E. Volchkov, Victoria Wahl-Jensen.
Members of the ICTV Mononegavirales Study Group who coauthored this manuscript: Ralf G. Dietzgen, Jens H. Kuhn, Gael Kurath, Bertus K. Rima, Norbert Nowotny, Andrew J. Easton, Dennis Rubbenstroth, Nikos Vasilakis, Peter J. Walker.

Members of the ICTV Nyamiviridae Study Group who coauthored this manuscript: Ralf G. Dietzgen, Elodie Ghedin, Dàohóng Jiāng, Jens H. Kuhn, Nikos Vasilakis, David Wang.

Members of the ICTV Paramyxoviridae Study Group who coauthored this manuscript: Peter L. Collins, Andrew J. Easton, Ron A. M. Fouchier, Gael Kurath, Robert A. Lamb, Andrea Maisner, Bertus K. Rima, Paul Rota, Benhur Lee, Ming Li, Lin-Fa Wang.

Members of the ICTV Rhabdoviridae Study Group who coauthored this manuscript: Kim R. Blasdell, Charles H. Calisher, Ralf G. Dietzgen, Hideki Kondo, Gael Kurath, David M. Stone, Robert B. Tesh, Noël Tordo, Nikos Vasilakis, Peter J. Walker, Anna E. Whitfield. 


\section{Compliance with ethical standards}

The views and conclusions contained in this document are those of the authors and should not be interpreted as necessarily representing the official policies, either expressed or implied, of the US Department of the Army, the US Department of Defense, the US Department of Health and Human Services, the Department of Homeland Security (DHS) Science and Technology Directorate (S\&T) or of the institutions and companies affiliated with the authors. In no event shall any of these entities have any responsibility or liability for any use, misuse, inability to use, or reliance upon the information contained herein. The US departments do not endorse any products or commercial services mentioned in this publication.

Funding This work was supported in part through Battelle Memorial Institute's prime contract with the US National Institute of Allergy and Infectious Diseases (NIAID) under Contract No. HHSN272200700016I. An employee of Battelle Memorial Institute is: A. Bochnowski. A subcontractor to Battelle Memorial Institute who performed this work is: J.H.K., an employee of Tunnell Government Services, Inc. This work was also funded in part under Contract No. HSHQDC-15-C-00064 awarded by DHS S\&T for the management and operation of the National Biodefense Analysis and Countermeasures Center (NBACC), a Federally Funded Research and Development Center (V.W.-J.); and National Institutes of Health (NIH) contract HHSN272201000040I/HHSN27200004/D04 and Grant R24AI120942 (N.V., R.B.T.). Y.B. was supported by the Intramural Research Program of the NIH, National Library of Medicine.

Conflict of interest The authors have no conflicts of interest.

Ethical approval This article does not contain any studies with human participants or animals performed by any of the authors.

\section{References}

1. Adams MJ, Lefkowitz EJ, King AM, Carstens EB (2014) Ratification vote on taxonomic proposals to the International Committee on Taxonomy of Viruses (2014). Arch Virol 159:2831-2841

2. Afonso $\mathrm{CL}$, Amarasinghe GK, Bányai K, Bào Y, Basler CF, Bavari S, Bejerman N, Blasdell KR, Briand F-X, Briese T, Bukreyev A, Calisher CH, Chandran K, Chéng J, Clawson AN, Collins PL, Dietzgen RG, Dolnik O, Domier LL, Dürrwald R, Dye JM, Easton AJ, Ebihara H, Farkas SL, Freitas-Astúa J, Formenty P, Fouchier RA, Fù Y, Ghedin E, Goodin MM, Hewson R, Horie M, Hyndman TH, Jiāng D, Kitajima EW, Kobinger GP, Kondo H, Kurath G, Lamb RA, Lenardon S, Leroy EM, Li C-X, Lin X-D, Liú L, Longdon B, Marton S, Maisner A, Mühlberger E, Netesov SV, Nowotny N, Patterson JL, Payne SL, Paweska JT, Randall RE, Rima BK, Rota P, Rubbenstroth D, Schwemmle M, Shi M, Smither SJ, Stenglein MD, Stone DM, Takada A, Terregino C, Tesh RB, Tian J-H, Tomonaga K, Tordo N, Towner JS, Vasilakis N, Verbeek M, Volchkov VE, Wahl-Jensen V, Walsh JA, Walker PJ, Wang D, Wang L-F, Wetzel T, Whitfield AE, Xiè JT, Yuen K-Y, Zhang Y-Z, Kuhn JH (2016) Taxonomy of the order Mononegavirales: update 2016. Arch Virol 161:2351-2360

3. Albariño CG, Foltzer M, Towner JS, Rowe LA, Campbell S, Jaramillo CM, Bird BH, Reeder DM, Vodzak ME, Rota P, Metcalfe MG, Spiropoulou CF, Knust B, Vincent JP, Frace MA, Nichol ST, Rollin PE, Ströher U (2014) Novel paramyxovirus associated with severe acute febrile disease, South Sudan and Uganda, 2012. Emerg Infect Dis 20:211-216
4. Allison AB, Mead DG, Palacios GF, Tesh RB, Holmes EC (2014) Gene duplication and phylogeography of North American members of the Hart Park serogroup of avian rhabdoviruses. Virology 448:284-292

5. Baker KS, Todd S, Marsh GA, Crameri G, Barr J, Kamins AO, Peel AJ, Yu M, Hayman DT, Nadjm B, Mtove G, Amos B, Reyburn H, Nyarko E, Suu-Ire R, Murcia PR, Cunningham AA, Wood JL, Wang L-F (2013) Novel, potentially zoonotic paramyxoviruses from the African straw-colored fruit bat Eidolon helvum. J Virol 87:1348-1358

6. Binger T, Annan A, Drexler JF, Müller MA, Kallies R, Adankwah E, Wollny R, Kopp A, Heidemann H, Dei D, Agya-Yao FC, Junglen S, Feldt T, Kurth A, Oppong S, Adu-Sarkodie Y, Drosten C (2015) A novel rhabdovirus isolated from the straw-colored fruit bat Eidolon helvum, with signs of antibodies in swine and humans. J Virol 89:4588-4597

7. Bishop DHL, Pringle CR (1995) Order Mononegavirales. In: Murphy FA, Fauquet CM, Bishop DHL, Ghabrial SA, Jarvis AW, Martelli GP, Mayo MA, Summers MD (eds) Virus TaxonomySixth Report of the International Committee on Taxonomy of Viruses/Archives of Virology Supplement 10. Springer-Verlag, Vienna, Austria, pp 265-267

8. Blasdell KR, Voysey R, Bulach DM, Trinidad L, Tesh RB, Boyle DB, Walker PJ (2012) Malakal virus from Africa and Kimberley virus from Australia are geographic variants of a widely distributed ephemerovirus. Virology 433:236-244

9. Blasdell KR, Widen SG, Diviney SM, Firth C, Wood TG, Guzman H, Holmes EC, Tesh RB, Vasilakis N, Walker PJ (2014) Koolpinyah and Yata viruses: two newly recognised ephemeroviruses from tropical regions of Australia and Africa. Vet Microbiol 174:547-553

10. Blasdell KR, Guzman H, Widen SG, Firth C, Wood TG, Holmes EC, Tesh RB, Vasilakis N, Walker PJ (2015) Ledantevirus: a proposed new genus in the Rhabdoviridae has a strong ecological association with bats. Am J Trop Med Hyg 92:405-410

11. Burroughs AL, Tachedjian M, Crameri G, Durr PA, Marsh GA, Wang L-F (2015) Complete genome sequence of Teviot paramyxovirus, a novel rubulavirus isolated from fruit bats in Australia. Genome Announc 3:e00115-e00177

12. Chua KB, Wang L-F, Lam SK, Eaton BT (2002) Full length genome sequence of Tioman virus, a novel paramyxovirus in the genus Rubulavirus isolated from fruit bats in Malaysia. Arch Virol 147:1323-1348

13. Contreras MA, Eastwood G, Guzman H, Popov V, Savit C, Uribe S, Kramer LD, Wood TG, Widen SG, Fish D, Tesh RB, Vasilakis N, Walker PJ (2017) Almendravirus: a proposed new genus of rhabdoviruses isolated from mosquitoes in tropical regions of the Americas. Am J Trop Med Hyg 96:100-109

14. de Almeida Medeiros DB, Diniz Júnior JAP, Cardoso JF, Silva SP, da Silva DEA, de Oliveira LF, é Vasconcelos JM, Chiang JO, Dias AA, Nunes MR, de Vianez Júnior SGJL, Vasconcelos PFC (2014) Nearly complete genome sequence of Curionopolis virus, a Culicoides-related rhabdovirus isolated in the Brazilian Amazon region. Genome Announc 2:e01114-e01158

15. Diniz JAP, Nunes MRT, Travassos da Rosa APA, Cruz ACR, de Souza W, Medeiros DBA, Chiang JO, Vasconcelos PFC (2006) Characterization of two new rhabdoviruses isolated from midges (Culicoides spp) in the Brazilian Amazon: proposed members of a new genus, Bracorhabdovirus. Arch Virol 151:2519-2527

16. Drexler JF, Corman VM, Müller MA, Maganga GD, Vallo P, Binger T, Gloza-Rausch F, Cottontail VM, Rasche A, Yordanov S, Seebens A, Knornschild M, Oppong S, Adu Sarkodie Y, Pongombo C, Lukashev AN, Schmidt-Chanasit J, Stöcker A, Carneiro AJ, Erbar S, Maisner A, Fronhoffs F, Buettner R, Kalko EKV, Kruppa T, Franke CR, Kallies R, Yandoko ERN, Herrler G, Reusken C, Hassanin A, Krüger DH, Matthee S, Ulrich RG, 
Leroy EM, Drosten C (2012) Bats host major mammalian paramyxoviruses. Nat Commun 3:796

17. Easton AJ, Pringle CR (2011) Order Mononegavirales. In: King AMQ, Adams MJ, Carstens EB, Lefkowitz EJ (eds) Virus Taxonomy-Ninth Report of the International Committee on Taxonomy of Viruses. Elsevier/Academic Press, London, pp 653-657

18. Ghedin E, Rogers MB, Widen SG, Guzman H, Travassos da Rosa AP, Wood TG, Fitch A, Popov V, Holmes EC, Walker PJ, Vasilakis N, Tesh RB (2013) Kolente virus, a rhabdovirus species isolated from ticks and bats in the Republic of Guinea. J Gen Virol 94:2609-2615

19. Goraichuk I, Sharma P, Stegniy B, Muzyka D, Pantin-Jackwood MJ, Gerilovych A, Solodiankin O, Bolotin V, Miller PJ, Dimitrov KM, Afonso CL (2016) Complete genome sequence of an avian paramyxovirus representative of putative new serotype 13 . Genome Announc 4:e00716-e00729

20. Grard G, Fair JN, Lee D, Slikas E, Steffen I, Muyembe J-J, Sittler T, Veeraraghavan N, Ruby JG, Wang C, Makuwa M, Mulembakani P, Tesh RB, Mazet J, Rimoin AW, Taylor T, Schneider BS, Simmons G, Delwart E, Wolfe ND, Chiu CY, Leroy EM (2012) A novel rhabdovirus associated with acute hemorrhagic fever in Central Africa. PLoS Pathog 8:e1002924

21. Gubala AJ, Proll DF, Barnard RT, Cowled CJ, Crameri SG, Hyatt AD, Boyle DB (2008) Genomic characterisation of Wongabel virus reveals novel genes within the Rhabdoviridae. Virology 376:13-23

22. Higgins CM, Bejerman N, Li M, James AP, Dietzgen RG, Pearson MN, Revill PA, Harding RM (2016) Complete genome sequence of Colocasia bobone disease-associated virus, a putative cytorhabdovirus infecting taro. Arch Virol 161:745-748

23. Hoffmann B, Tappe D, Höper D, Herden C, Boldt A, Mawrin C, Niederstraßer O, Müller T, Jenckel M, van der Grinten E, Lutter C, Abendroth B, Teifke JP, Cadar D, Schmidt-Chanasit J, Ulrich RG, Beer M (2015) A variegated squirrel bornavirus associated with fatal human encephalitis. N Engl J Med 373:154-162

24. Karamendin K, Kydyrmanov A, Seidalina A, Asanova S, Sayatov M, Kasymbekov E, Khan E, Daulbayeva K, Harrison SM, Carr IM, Goodman SJ, Zhumatov K (2016) Complete genome sequence of a novel avian paramyxovirus (APMV-13) isolated from a wild bird in Kazakhstan. Genome Announc 4:e00116e00167

25. Kuhn JH, Bekal S, Caì Y, Clawson AN, Domier LL, Herrel M, Jahrling PB, Kondo H, Lambert KN, Mihindukulasuriya KA, Nowotny N, Radoshitzky SR, Schneider U, Staeheli P, Suzuki N, Tesh RB, Wang D, Wang L-F, Dietzgen RG (2013) Nyamiviridae: proposal for a new family in the order Mononegavirales. Arch Virol 158:2209-2226

26. Lau SKP, Woo PCY, Wong BHL, Wong AYP, Tsoi H-W, Wang M, Lee P, Xu H, Poon RWS, Guo R, Li KSM, Chan K-H, Zheng B-J, Yuen K-Y (2010) Identification and complete genome analysis of three novel paramyxoviruses, Tuhoko virus 1,2 and 3, in fruit bats from China. Virology 404:106-116

27. Lauck M, Yú SQ, Caì Y, Hensley LE, Chiu CY, O'Connor DH, Kuhn JH (2015) Genome sequence of Bivens Arm virus, a tibrovirus belonging to the species Tibrogargan virus (Mononegavirales: Rhabdoviridae). Genome Announc 3:e00015-e00089

28. Li C-X, Shi M, Tian J-H, Lin X-D, Kang Y-J, Chen L-J, Qin X-C, $\mathrm{Xu}$ J, Holmes EC, Zhang Y-Z (2015) Unprecedented genomic diversity of RNA viruses in arthropods reveals the ancestry of negative-sense RNA viruses. Elife 4:e05378

29. McAllister J, Gauci PJ, Mitchell IR, Boyle DB, Bulach DM, Weir RP, Melville LF, Davis SS, Gubala AJ (2014) Genomic characterisation of Almpiwar virus, Harrison Dam virus and Walkabout Creek virus; three novel rhabdoviruses from northern Australia. Virology Reports 3-4:1-17
30. Ng TF, Driscoll C, Carlos MP, Prioleau A, Schmieder R, Dwivedi B, Wong J, Cha Y, Head S, Breitbart M, Delwart E (2013) Distinct lineage of vesiculovirus from big brown bats, United States. Emerg Infect Dis 19:1978-1980

31. Pfeffer M, Dilcher M, Tesh RB, Hufert FT, Weidmann M (2013) Genetic characterization of Yug Bogdanovac virus. Virus Genes 46:201-202

32. Pringle CR (1991) Order Mononegavirales. In: Francki RIB, Fauquet CM, Knudson DL, Brown F (eds) Classification and Nomenclature of Viruses-Fifth Report of the International Committee on Taxonomy of Viruses/Archives of Virology Supplementum 2. Springer-Verlag, Vienna, Austria, pp 239-241

33. Pringle CR, Alexander DJ, Billeter MA, Collins PL, Kingsbury DW, Lipkind MA, Nagai Y, Orvell C, Rima B, Rott R, ter Meulen V (1991) The order Mononegavirales. Arch Virol 117:137-140

34. Pringle CR (1996) Virus taxonomy 1996-a bulletin from the Xth International Congress of Virology in Jerusalem. Arch Virol 141:2251-2256

35. Pringle CR (1997) The order Mononegavirales - current status. Arch Virol 142:2321-2326

36. Pringle CR (2000) Order Mononegavirales. In: van Regenmortel MHV, Fauquet CM, Bishop DHL, Carstens EB, Estes MK, Lemon SM, Maniloff J, Mayo MA, McGeoch DJ, Pringle CR, Wickner RB (eds) Virus Taxonomy-Seventh Report of the International Committee on Taxonomy of Viruses. Academic Press, San Diego, California, USA, pp 525-530

37. Pringle CR (2005) Order Mononegavirales. In: Fauquet CM, Mayo MA, Maniloff J, Desselberger U, Ball LA (eds) Virus Taxonomy-Eighth Report of the International Committee on Taxonomy of Viruses. Elsevier/Academic Press, San Diego, California, USA, pp 609-614

38. Sakai K, Hagiwara K, Omatsu T, Hamasaki C, Kuwata R, Shimoda H, Suzuki K, Endoh D, Nagata N, Nagai M, Katayama Y, Oba M, Kurane I, Saijo M, Morikawa S, Mizutani T, Maeda K (2015) Isolation and characterization of a novel rhabdovirus from a wild boar (Sus scrofa) in Japan. Vet Microbiol 179:197-203

39. Stremlau MH, Andersen KG, Folarin OA, Grove JN, Odia I, Ehiane PE, Omoniwa O, Omoregie O, Jiang P-P, Yozwiak NL, Matranga CB, Yang X, Gire SK, Winnicki S, Tariyal R, Schaffner SF, Okokhere PO, Okogbenin S, Akpede GO, Asogun DA, Agbonlahor DE, Walker PJ, Tesh RB, Levin JZ, Garry RF, Sabeti PC, Happi CT (2015) Discovery of novel rhabdoviruses in the blood of healthy individuals from West Africa. PLoS Negl Trop Dis 9:e0003631

40. Van Regenmortel MH, Burke DS, Calisher CH, Dietzgen RG, Fauquet CM, Ghabrial SA, Jahrling PB, Johnson KM, Holbrook MR, Horzinek MC, Keil GM, Kuhn JH, Mahy BW, Martelli GP, Pringle C, Rybicki EP, Skern T, Tesh RB, Wahl-Jensen V, Walker PJ, Weaver SC (2010) A proposal to change existing virus species names to non-Latinized binomials. Arch Virol 155:1909-1919

41. Vasilakis N, Widen S, Mayer SV, Seymour R, Wood TG, Popov V, Guzman H, Travassos da Rosa APA, Ghedin E, Holmes EC, Walker PJ, Tesh RB (2013) Niakha virus: a novel member of the family Rhabdoviridae isolated from phlebotomine sandflies in Senegal. Virology 444:80-89

42. Vasilakis N, Widen S, Travassos da Rosa APA, Wood TG, Walker PJ, Holmes EC, Tesh RB (2013) Malpais Spring virus is a new species in the genus Vesiculovirus. Virol J 10:69

43. Vasilakis N, Castro-Llanos F, Widen SG, Aguilar PV, Guzman H, Guevara C, Fernandez R, Auguste AJ, Wood TG, Popov V, Mundal K, Ghedin E, Kochel TJ, Holmes EC, Walker PJ, Tesh RB (2014) Arboretum and Puerto Almendras viruses: two novel rhabdoviruses isolated from mosquitoes in Peru. J Gen Virol 95:787-792 
44. Walker PJ, Firth C, Widen SG, Blasdell KR, Guzman H, Wood TG, Paradkar PN, Holmes EC, Tesh RB, Vasilakis N (2015) Evolution of genome size and complexity in the Rhabdoviridae. PLoS Pathog 11:e1004664

45. Wang F, Fang Q, Wang B, Yan Z, Hong J, Bao Y, Kuhn JH, Werren JH, Song Q, Ye G (2017) A novel negative-stranded RNA virus mediates sex ratio in its parasitoid host. PLoS Pathog 3:e1006201
46. World Organisation for Animal Health (OIE) (2010) Chapter 10.13. Newcastle disease. Terrestrial Animal Health Code, Paris, France. http://web.oie.int/eng/normes/mcode/en_chapitre_ 1.10.13.pdf

47. Yamamoto E, Ito H, Tomioka Y, Ito T (2015) Characterization of novel avian paramyxovirus strain APMV/Shimane67 isolated from migratory wild geese in Japan. J Vet Med Sci 77:1079-1085 